\title{
New oral anticoagulants: just a new therapeutic option or a real breakthrough?
}

\author{
Giancarlo Agnelli $\cdot$ Cecilia Becattini
}

Received: 23 September 2009/Accepted: 23 September 2009/Published online: 30 October 2009

(C) SIMI 2009

The number of anticoagulant agents currently available for clinical use is relatively limited. This is immediately clear when the number of the available anticoagulants is matched up to that of antimicrobial or the anti-inflammatory agents or the drugs used to treat arterial hypertension. Despite their reduced number, treatment with the available anticoagulant agents has been associated with an outstanding improvement of clinical care. Unfractionated heparin, several low-molecular weight heparins and fondaparinux reduce by more than $60 \%$ the rate of venous thromboembolism in patients undergoing major orthopedic or cancer surgery and in high-risk medical patients. Vitamin $\mathrm{K}$ antagonists reduce by more than $90 \%$ the risk of recurrence in patients with deep vein thrombosis or pulmonary embolism and by about $60 \%$ the risk of stroke in patients with atrial fibrillation. Bivalirudin, a parenteral thrombin inhibitor, has been shown to be effective and safe in patients with acute coronary syndromes who had a stent implanted. Given these achievements, it could be easy to assume that the currently available agents, if properly used, are able to meet most of the clinical needs in the antithrombotic therapy. However, the currently available agents have several limitations. Unfractionated or lowmolecular weight heparin, fondaparinux or bivalirudin require parenteral administration and are cumbersome to be used in the so-called "chronic indications", that is to say, those requiring a prolonged administration. The oral vitamin $\mathrm{K}$ antagonists are associated with a remarkable individual variability in the dose-response and therefore

G. Agnelli $(\bowtie) \cdot$ C. Becattini

Medicina Interna e Cardiovascolare-Stroke Unit,

Dipartimento di Medicina Interna, Università di Perugia,

Via G. Dottori 1, 06129 Perugia, Italy

e-mail: agnellig@unipg.it require relatively frequent laboratory monitoring for dose adjustment. It makes clinical sense to anticipate that an oral agent at least as effective and safe as the vitamin $\mathrm{K}$ antagonists (but possibly more) to be given at fixed doses without laboratory monitoring would be a real breakthrough in the current clinical practice. Indeed, prophylaxis of postoperative thromboembolism in high-risk patients could be easily extended, long-term treatment of venous thromboembolism and prophylaxis of stroke in patients with atrial fibrillation could be made less cumbersome. In patients with atrial fibrillation treatment would be made available for that $50 \%$ of patients to whom anticoagulant treatment is denied for the impracticality of the vitamin $\mathrm{K}$ antagonists.

In the last 10 years a number of new oral agents have entered the procedures of the clinical evaluation. The clinical development of most of these agents did not go beyond the phase II (dose-finding) studies, but some among the survivors of such a tremendous selection process reached or are about to reach the clinical use. The clinical development of an antithrombotic agent follows a secure pathway which a critical mind could also see as a sort of imagination-free stereotype. Indeed, almost all the new agents are first tested in the prevention of venous thromboembolism in major orthopedic surgery and, only after they have been shown to be effective and safe in this indication, are further evaluated in the treatment of venous thromboembolism and in the prevention of stroke in patients with atrial fibrillation. Prevention of venous thromboembolism in major orthopedic surgery is the preferred initial step for Phase II and Phase III studies as it is associated with a high and predictable event rate, a reliable measurability of the study outcome (deep vein thrombosis as assessed by venography) and a relatively careful assessment of agent-related bleeding. Once the clinical development has been completed, the 
availability of an effective and safe oral agent would certainly facilitate the extension of antithrombotic prophylaxis after hospital discharge thus avoiding the late events associated with high-risk orthopedic surgery. However, an oral antithrombotic agent is expected to produce the most remarkable changes in the long-term treatment of venous thromboembolism and in the prevention of stroke in patients with atrial fibrillation.

The most promising among the new oral antithrombotic agents are selective inhibitors of either factor Xa or thrombin. The available data on these two groups of antithrom-

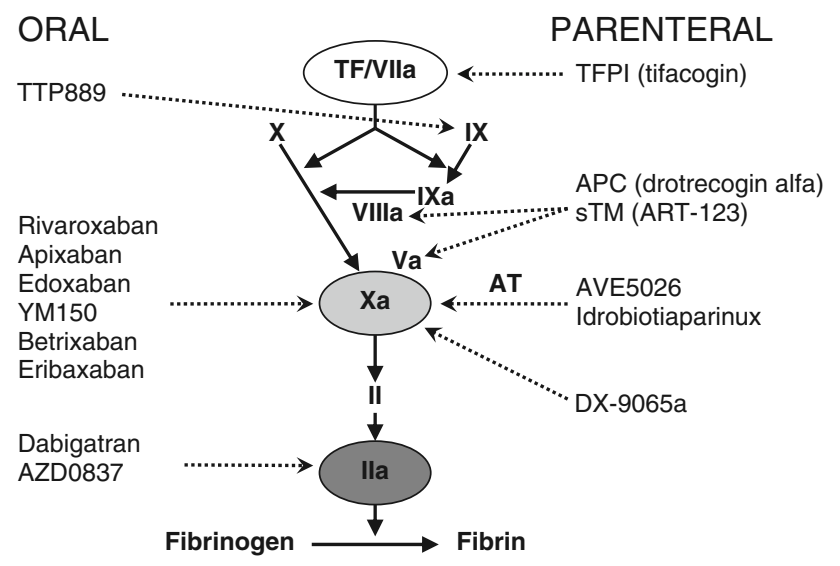

Fig. 1 Anticoagulant agents under clinical development

botic agents are brilliantly and clearly reviewed by Imberti et al. and Squizzato et al. in Internal and Emergency Medicine $[1,2]$. The biochemistry background and the pharmacological advantages associated with selectively targeting either factor Xa or thrombin are also reviewed. Indeed, the head-to-head publication of the two articles draws renewed attention to the debate about the relative benefit associated with the selective inhibition of factor Xa or thrombin. This debate started about 30 years ago and it is related to the dream of clinical pharmacologists and clinicians to dissociate the antithrombotic from the hemorrhagic effect of the anticoagulant agents. For many years the only way to address this issue was the separation of heparin fractions composed by chains with prevalent antithrombin or anti-Xa activity. The introduction of molecular biology allowed the sequencing of the active (catalytic) site of the single coagulation factors and the production of antithrombotic agents exclusively directed toward such an active site. The ongoing clinical trials with the oral anti-Xa or thrombin inhibitors will provide further interest to the debate about their relative value. However, it should be taken into account that, in addition to the intrinsic anticoagulant activity, the clinical value of an agent is also related to its clinical pharmacology and to the identification of the optimal doses in properly performed Phase II studies (Fig. 1).
In the absence of clinical trials directly comparing an oral thrombin inhibitor with an oral anti-Xa inhibitor, it is probably unfair to make a definitive judgment about the relative efficacy and safety of these agents. Furthermore, the comparison is probably premature as the clinical development of the new agents should be seen today as a work in progress. If the reader focuses on the efficacy, he/ she will find undoubtedly impressive the consistency of the results of the studies with the anti-Xa inhibitor rivaroxaban. If the reader focuses on the safety, he/she will find reassuring the results achieved with the thrombin inhibitor dabigatran. The dispute about the clinical benefit associated with these two agents cannot ignore the results of the recently reported RE-LY study [3]. This study showed a remarkable clinical benefit of dabigatran over warfarin in the prevention of stroke in patients with atrial fibrillation. Indeed, dabigatran was associated with a reduction of the risk of stroke without excessive bleeding. The results of the currently ongoing studies with the anti-Xa inhibitors rivaroxaban and apixaban in the prevention of stroke in patients with atrial fibrillation are anxiously awaited.

Whether at the end of the ongoing studies we will have a particular antithrombotic agent as the winner for all the clinical indications is a difficult guess. Indeed, it is quite likely that different agents will be the winners in different clinical settings. It is even possible that patients with the same thromboembolic disease will receive different antithrombotic agent based on their clinical features or the individual risk for thrombotic or bleeding events.

Are the new oral antithrombotic agents just a new therapeutic option or a real breakthrough? It is quite likely that this question will be answered only after an extensive clinical use of these agents. With the new oral agents no laboratory monitoring will be required and this can be certainly regarded as a remarkable improvement in the management of long-term anticoagulant treatment. On the other hand, it is fair to anticipate some concerns related to the long-term use of the new oral antithrombotic agents. The administration without monitoring could probably result in reduced patient compliance, self-adjustment of the dose, less attention to comedication and, in general, less careful clinical follow-up. Therefore, the new oral antithrombotic agents will be a real therapeutic breakthrough only if less careful patient care will not be the trade-off for improved practicality.

Conflict of interest statement The authors declare that they have no conflict of interest related to the publication of this manuscript.

\section{References}

1. Imberti D, Dall'Asta C, Giorgi Pierfranceschi M (2009) Oral factor Xa inhibitors for thromboprophylaxis in major orthopedic surgery: a review. Intern Emerg Med J. doi:10.1007/s11739-009-0293-9 
2. Squizzato A, Dentali F, Steidl L, Ageno W (2009) New direct thrombin inhibitors. Intern Emerg Med J. doi:10.1007/s11739009-0314-8

3. Connolly SJ, Ezekowitz MD, Yusuf S, Eikelboom J, Oldgren J, Parekh A, Pogue J, Reilly PA, Themeles E, Varrone J, Wang S,
Alings M, Xavier D, Zhu J, Diaz R, Lewis BS, Darius H, Diener HC, Joyner CD, Wallentin L, RE-LY Steering Committee and Investigators (2009) Dabigatran versus warfarin in patients with atrial fibrillation. N Engl J Med 361:1139-1151 